\title{
Sociodemographic, behavioral, and substance use correlates of reckless driving in the United States: Findings from a national Sample
}

\author{
Michael G. Vaughn ${ }^{\mathrm{a}, *}$, Rebecca S. Define ${ }^{\mathrm{b}}$, Matt DeLisi ${ }^{\mathrm{c}}$, Brian E. Perron ${ }^{\mathrm{d}}$, Kevin M. Beaver ${ }^{\mathrm{e}}$, \\ Qiang Fu ${ }^{\text {f }}$, Matthew O. Howard ${ }^{\mathrm{g}}$

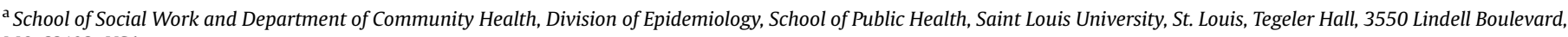 \\ MO 63103, USA \\ ${ }^{\mathrm{b}}$ Research Division, Queen of Peace Residential Treatment Center, St. Louis, MO, USA \\ ${ }^{c}$ Criminology and Criminal Justice Studies, Department of Sociology, Iowa State University, Ames, IA, USA \\ d School of Social Work, University of Michigan, Ann Arbor, MI, USA \\ e College of Criminology and Criminal Justice, Florida State University, Tallahassee, FL, USA \\ ${ }^{\mathrm{f}}$ Department of Biostatistics, School of Public Health, Saint Louis University, St. Louis, MO, USA \\ ${ }^{\mathrm{g}}$ School of Social Work, University of North Carolina, Chapel Hill, NC, USA
}

\section{A R T I C L E I N F O}

\section{Article history:}

Received 14 May 2010

Received in revised form

29 June 2010

Accepted 29 June 2010

\section{Keywords:}

Antisocial behavior

Comorbidity

Drinking

Driving

Reckless driving

\begin{abstract}
A B S T R A C T
This study examined the sociodemographic, behavioral, psychiatric, and substance use correlates of three forms of reckless driving using a nationally representative sample of U.S. adults. Participants were 43,093 adults from the National Epidemiologic Survey on Alcohol and Related Conditions (NESARC). Interviewers administered the Alcohol Use Disorder and Associated Disabilities Interview Schedule - DSM-IV version (AUDADIS-IV). This measure provides extensive sociodemographic data as well as diagnoses for mood, anxiety, personality, and substance use disorders. Reckless driving was significantly associated with male gender, lower levels of income, being born in the U.S., and numerous forms of antisocial behaviors. Fully adjusted models revealed significant effects with respect to substance use disorders across categories of reckless drivers with those having their licenses revoked or suspended being particularly more likely to be diagnosed with antisocial (AOR $=3.35,95 \% \mathrm{CI}=2.54,4.42$ ) and paranoid personality disorder $(\mathrm{AOR}=1.56,95 \% \mathrm{CI}=1.07,2.29)$. All three reckless driving groups were more likely to have a family history of antisocial behavior than non-reckless drivers. Study findings provide information from which targeted behavioral interventions can be applied.
\end{abstract}

(c) 2010 Elsevier Ltd. All rights reserved.
Reckless driving, defined as wanton disregard of traffic laws which poses a threat to traffic safety, is a significant social and public health problem in the U.S. and worldwide. In higher-income, developed nations, for example, road traffic injuries are the leading cause of death for persons ages 5 to 14 and 15 to 44 . Road traffic injuries-many which are the direct result of reckless driving-constitute the 10th leading cause of death globally and the 9th leading contributor to disease burden (Krug et al., 2000). In the U.S., motor vehicle accidents are the leading cause of death for persons ages 1 to 34 and result in economic costs in excess of $\$ 230$ billion (Centers for Disease Control and Prevention,; Blincoe et al., 2002). Epidemiological studies from multiple nations indicate that the strongest determinant of traffic-related deaths, injuries, and economic costs is reckless driving particularly driving while under

\footnotetext{
* Corresponding author. Tel.: +1 314977 2718; fax: +1 3149772731.

E-mail address: mvaughn9@slu.edu (M.G. Vaughn).
}

the influence of alcohol (Borges et al., 2004; Nabi et al., 2005; Villaveces et al., 2003). In addition to these costs reckless driving can also have pernicious psychological consequences for victims and perpetrators including increased stress (De Vries et al., 1999; Ursano et al., 1999), PTSD symptoms (Keppel-Benson et al., 2002), and major depression one-year following a motor vehicle injury (Blincoe et al., 2002; Blanchard et al., 1995; Donovan and Marlatt, 1982).

\section{Substance use and reckless driving}

An important factor in reckless driving is alcohol and drug use. Of the 37,261 individuals who were killed in traffic crashes in 2008, 32\% (about 12,000) died as a result of alcohol impairment with blood alcohol concentration exceeding $0.08 \%$ (Trends in Alcohol Related Fatalities,). Alcohol-related motor vehicle accidents accounted for $22 \%$ of total economic costs due to motor vehicle accidents and $46 \%$ of fatality-related costs (Blincoe et al., 2002). Illicit drug use is involved in $5-25 \%$ of motor vehicle 
accidents; the most commonly detected drugs in impaired drivers were cannabis, followed by benzodiazepines, cocaine and other stimulants, and opioids (Kelly et al., 2004). In a comparative study of a New Mexico sample of convicted drunk drivers and participants in the National Comorbidity Survey, $85 \%$ of females and $91 \%$ of males in New Mexico had a lifetime alcohol use disorder (Lapham et al., 2001), prevalence rates that were significantly higher than the National Comorbidity Survey which reported rates of $22 \%$ of females and $44 \%$ of males, respectively. Of DWI offenders with alcohol use disorders, $50 \%$ of women and $33 \%$ of men had at least one additional psychiatric disorder (Lapham et al., 2001). In addition, $32 \%$ of women in the study and $38 \%$ of men had a drug use disorder compared to $16 \%$ and $21 \%$ respectively on the National Comorbidity Survey. It was also found that depression was highly correlated with motor vehicle accidents in men with a history of alcohol dependence (Donovan et al., 1983). Despite these results, there are relatively few systematic findings on the prevalence and correlates of reckless drivers and their comorbid conditions. This is unfortunate given that the economic costs and adverse health consequences of reckless driving are substantial. Although it is not surprising that reckless driving is associated with substance use disorders and antisocial behavior, few studies have documented the specific quantitative nature of these variables in conjunction other psychiatric disorders and sociodemographic factors.

\section{Study purpose}

The present study sought to fill in the gap in the literature on reckless drivers by examining sociodemographic, behavioral, psychiatric, and substance use correlates of reckless driving using a nationally representative sample of U.S. adults. The primary aims were to 1) compare adults with a lifetime history of reckless driving to individuals without such a history across sociodemographic variables, childhood and adult antisocial behaviors, and lifetime mood, anxiety, substance use, and personality disorders, and 2) to estimate the magnitude of associations between these variables and severity of reckless driving history in controlled multivariate analyses. Two hypotheses were tested: 1 ) reckless driving will be positively associated with substance use disorders and antisocial behavior even after controlling for sociodemographic characteristics and lifetime psychiatric disorders, and 2) severity of reckless driving history will be positively associated with prevalence and severity of substance use and antisocial behavior.

\section{Method}

\subsection{Participants}

Study findings are based on data from the 2001-2002 National Epidemiologic Survey on Alcohol and Related Conditions (NESARC). NESARC is a nationally representative sample of 43,093 non-institutionalized U.S. residents aged 18 years and older. (Grant et al., 2003). The survey gathered background data and extensive information about a wide range of behaviors. NESARC is the largest comorbidity survey to date collecting diagnostic information from individuals living in households and group settings such as shelters, college dormitories, and group homes in all 50 states and the District of Columbia. NESARC utilized a multistage cluster sampling design, oversampling young adults, Hispanics, and African-Americans in the interest of obtaining reliable statistical estimation in these subpopulations, and to ensure appropriate representation of racial/ethnic subgroups. The overall response rate was $81 \%$. Data were weighted at the individual and household levels to adjust for oversampling and non-response on demographic variables (i.e., age, race/ethnicity, sex, region, and place of residence). Data were also adjusted to be representative (based on region, age, race, and ethnicity) of the U.S. adult population as assessed during the 2000 Census. Study participants provided fully informed consent. The U.S. Census Bureau and the U.S. Office of Management and Budget approved the research protocol and informed consent procedures.

\subsection{Diagnostic Assessment and sociodemographic measures}

Data were collected through face-to-face structured psychiatric interviews conducted by U.S. Census workers trained by the National Institute on Alcohol Abuse and Alcoholism and U.S. Census Bureau. Interviewers administered the Alcohol Use Disorder and Associated Disabilities Interview Schedule - DSMIV version (AUDADIS-IV), which in addition to extensive background and sociodemographic data provides diagnoses for mood, anxiety, personality, and substance use disorders. The AUDADISIV has shown good-to-excellent reliability in assessing alcohol and drug use in the general population. (Grant et al., 1995; Hasin et al., 1997).

The lifetime prevalence of reckless driving was assessed with three items embedded in the antisocial behavior interview module. All NESARC participants were asked the following questions: In your entire life, "did you ever do things that could easily hurt you or someone else like speeding or driving after too much to drink?", "did you ever get more than 3 tickets for reckless/ careless driving, speeding, or causing an accident?", "did you ever have drivers license suspended or revoked for moving violations?" NESARC respondents who did not answer yes to any of these three items were defined as non-reckless drivers. Respondents who answered "yes" to only the first item were defined as being episodic reckless/drinking-related, those answering 'yes' to item two (regardless of their response to item one) were defined as chronically reckless, and participants who responded yes to any item and item three or item three only were considered as chronic/ severely reckless. In terms of the psychometric properties of the antisocial behavior section, the test-retest reliability for the antisocial personality disorder diagnosis was adequate $(r=0.69)$ (Grant et al., 2003). The internal consistency reliability for the entire antisocial personality disorder criterion set was also good ( $\alpha=.86$ ) (Blanco et al., 2008).

Consistent with current conceptualizations of personality disorders (Grant et al., 2004a, 2004b; Goldstein et al., 2006), DSMIV personality disorder diagnoses reflected characteristic patterns of behavior accompanied by longstanding impairment and the exclusion of cases where substance use intoxication or withdrawal, other medication use, or physical illnesses could have contributed to reported Axis II personality disorder symptoms and indicators. In addition to antisocial personality disorder, other personality disorders assessed included avoidant, dependent obsessive-compulsive, paranoid, schizoid, and histrionic disorders. Family history of antisocial behavior based on any parental or sibling history of antisocial behavior was also assessed. Response categories for region of residence in U.S., urbanicity, race/ethnicity, sex, age, marital status, educational background, unemployment status, and individual and family income are listed in Table 1.

\subsection{Statistical analyses}

Weighted prevalence estimates and standard errors were computed using SUDAAN Version 9.0. (Research Triangle Institute, 2004). This software implements a Taylor series linearization to adjust standard errors of estimates for complex survey sampling 
Table 1

Sociodemographic characteristics of NESARC participants with a history of reckless driving, by severity of reckless driving history.

\begin{tabular}{|c|c|c|c|c|c|c|c|c|c|c|c|c|c|c|}
\hline \multirow[t]{2}{*}{ Characteristic } & \multicolumn{2}{|c|}{$\begin{array}{l}\text { No Reckless } \\
\text { Driving } \\
(N=33524)\end{array}$} & \multicolumn{2}{|c|}{$\begin{array}{l}\text { Episodic - } \\
\text { drinking related } \\
(N=3149)\end{array}$} & \multicolumn{2}{|c|}{ Chronic $(N=4212)$} & \multicolumn{2}{|c|}{$\begin{array}{l}\text { Chronic/Severe } \\
(N=1102)\end{array}$} & \multicolumn{2}{|c|}{$\begin{array}{l}\text { Episodic- } \\
\text { drinking } \\
\text { related }\end{array}$} & \multicolumn{2}{|c|}{ Chronic } & \multicolumn{2}{|c|}{ Chronic/Severe } \\
\hline & $\%$ & $\mathrm{CI}^{\mathrm{a}}$ & $\%$ & $\mathrm{CI}$ & $\%$ & $\mathrm{CI}$ & $\%$ & $\mathrm{CI}$ & $\mathrm{OR}^{\mathrm{b}}$ & $\mathrm{CI}$ & OR & $\mathrm{CI}$ & OR & $\mathrm{CI}$ \\
\hline \multicolumn{15}{|l|}{ Sex } \\
\hline Men & 41.46 & $40.70,42.23$ & 61.99 & $59.84,64.10$ & 72.80 & $71.30,74.25$ & 84.60 & $82.12,86.80$ & 2.41 & $2.18,2.66$ & 4.02 & $3.70,4.36$ & 8.51 & $7.05,10.26$ \\
\hline Women & 58.54 & $57.77,59.30$ & 38.01 & $35.90,40.16$ & 27.20 & $25.75,28.70$ & 15.40 & $13.20,17.88$ & 1.00 & & 1.00 & & 1.00 & \\
\hline \multicolumn{15}{|l|}{ Race } \\
\hline White & 68.71 & $65.15,72.07$ & 85.57 & $83.47,87.44$ & 74.46 & $71.75,76.99$ & 74.72 & $70.99,78.12$ & 1.90 & $1.56,2.32$ & 1.21 & $1.04,1.42$ & 1.42 & $1.03,1.95$ \\
\hline African American & 11.65 & $10.34,13.10$ & 4.76 & $3.96,5.70$ & 11.58 & $10.11,13.24$ & 10.17 & $8.40,12.27$ & 0.71 & $0.56,0.90$ & 1.13 & $0.95,1.35$ & 1.10 & $0.78,1.54$ \\
\hline Native American & 1.83 & $1.55,2.18$ & 2.92 & $2.27,3.76$ & 2.76 & $2.15,3.54$ & 4.18 & $2.75,6.30$ & 2.48 & $1.81,3.41$ & 1.60 & $1.20,2.15$ & 2.77 & $1.64,4.67$ \\
\hline Asian/Hawaiian/Indian & 7.04 & $5.95,8.32$ & 4.39 & $3.52,5.48$ & 4.55 & $3.77,5.49$ & 5.11 & $3.57,7.25$ & 0.72 & $0.48,1.07$ & 0.60 & $0.44,0.83$ & 0.33 & $0.15,0.73$ \\
\hline Hispanic & 12.60 & $10.13,15.55$ & 5.28 & $4.11,6.75$ & 9.41 & $7.51,11.72$ & 10.01 & $7.63,13.01$ & 1.00 & & 1.00 & & 1.00 & \\
\hline \multicolumn{15}{|l|}{ Nativity } \\
\hline Born in the U.S. & 82.97 & $79.25,86.14$ & 96.35 & $95.37,97.13$ & 92.50 & $90.81,93.91$ & 91.73 & $89.14,93.75$ & 3.87 & $3.03,4.95$ & 2.45 & $2.02,2.96$ & 2.10 & $1.40,3.14$ \\
\hline Born outside the U.S. & 17.03 & $13.86,20.75$ & 3.65 & $2.87,4.63$ & 7.50 & $6.09,9.19$ & 8.27 & $6.25,10.86$ & 1.00 & & 1.00 & & 1.00 & \\
\hline \multicolumn{15}{|l|}{ Age (years) } \\
\hline $65+$ & 18.89 & $18.05,19.76$ & 7.28 & $6.37,8.31$ & 7.51 & $6.69-8.42$ & 4.49 & $3.24,6.18$ & 0.28 & $0.24,0.32$ & 0.26 & $0.23,0.31$ & 0.12 & $0.08,0.18$ \\
\hline $50-64$ & 21.06 & $20.47,21.67$ & 21.56 & $19.88,23.34$ & 21.39 & $19.84,23.03$ & 18.26 & $15.80,21.00$ & 0.71 & $0.62,0.84$ & 0.74 & $0.65,0.84$ & 0.52 & $0.41,0.66$ \\
\hline $35-49$ & 29.51 & $28.81,30.23$ & 35.97 & $34.02,37.96$ & 36.43 & $34.61,38.29$ & 38.94 & $35.10,42.93$ & 0.91 & $0.82,1.02$ & 0.97 & $0.87,1.09$ & 0.90 & $0.73,1.11$ \\
\hline $18-34$ & 30.53 & $29.57,31.51$ & 35.20 & $33.09,37.36$ & 34.67 & $32.84,36.55$ & 38.31 & $34.55,42.23$ & 1.00 & & 1.00 & & 1.00 & \\
\hline \multicolumn{15}{|l|}{ Education } \\
\hline Less than High School & 16.40 & $15.35,17.50$ & 7.81 & $6.65-9.15$ & 14.87 & $13.58,16.26$ & 17.98 & $15.42,20.85$ & 0.62 & $0.52,0.75$ & 1.13 & $1.00,1.29$ & 1.59 & $1.28,1.97$ \\
\hline High School Graduate & 29.49 & $28.33,30.68$ & 25.02 & $22.84,27.34$ & 29.98 & $28.08,31.95$ & 34.34 & $30.88,37.97$ & 0.75 & $0.67,0.85$ & 1.03 & $0.93,1.13$ & 1.33 & $1.12,1.59$ \\
\hline Some College & 54.11 & $52.78,55.43$ & 67.17 & $64.75,69.50$ & 55.15 & $53.01,57.26$ & 47.69 & $44.01,51.39$ & 1.00 & & 1.00 & & 1.00 & \\
\hline \multicolumn{15}{|l|}{ Income } \\
\hline$<19,999$ & 24.77 & $23.75,25.82$ & 16.75 & $15.03,18.63$ & 20.22 & $18.69,21.85$ & 19.81 & $17.24,22.67$ & 0.94 & $0.80,1.10$ & 1.09 & $0.93,1.27$ & 1.11 & $0.83,1.47$ \\
\hline $20,000-34,999$ & 20.21 & $19.52,20.91$ & 17.09 & $15.48,18.82$ & 20.67 & $19.17,22.27$ & 24.42 & $21.17,27.98$ & 0.96 & $0.83,1.11$ & 1.15 & $1.00,1.32$ & 1.39 & $1.06,1.81$ \\
\hline $35,000-69,999$ & 31.54 & $30.85,32.24$ & 35.31 & $33.11,37.57$ & 34.04 & $32.28,35.85$ & 33.89 & $30.35,37.62$ & 0.99 & $0.88,1.12$ & 1.06 & $0.94,1.21$ & 1.12 & $0.88,1.42$ \\
\hline $70,000+$ & 23.48 & $22.07,24.95$ & 30.86 & $28.32,33.51$ & 25.06 & $22.92,27.33$ & 21.88 & $18.44,25.75$ & 1.00 & & 1.00 & & 1.00 & \\
\hline \multicolumn{15}{|l|}{ Marital Status } \\
\hline Never Married & 20.35 & $19.34,21.41$ & 21.86 & $19.93,23.92$ & 21.65 & $20.08,23.32$ & 22.67 & $19.85,25.76$ & 0.89 & $0.79,1.01$ & 0.81 & $0.71,0.92$ & 0.79 & $0.63-1.00$ \\
\hline Widowed/separated/divorced & 17.83 & $17.33,18.34$ & 13.92 & $12.63,15.32$ & 16.01 & $14.83,17.26$ & 19.66 & $17.25,22.31$ & 1.13 & $0.99,1.28$ & 1.28 & $1.15,1.41$ & 1.90 & $1.59,2.28$ \\
\hline Married/Cohabitating & 61.82 & $60.80,62.83$ & 64.23 & $62.05,66.35$ & 62.34 & $60.55,64.10$ & 57.67 & $54.43,60.86$ & 1.00 & & 1.00 & & 1.00 & \\
\hline \multicolumn{15}{|l|}{ Urbanicity } \\
\hline Urban & 30.35 & $25.65,35.49$ & 24.90 & $21.92,28.14$ & 27.09 & $24.11,30.30$ & 27.14 & $23.42,31.22$ & 0.93 & $0.82,1.05$ & 0.92 & $0.80,1.05$ & 0.93 & $0.75,1.14$ \\
\hline Rural & 69.65 & $64.51,74.35$ & 75.10 & $71.86,78.08$ & 72.91 & $69.70,75.89$ & 72.86 & $68.78,76.58$ & 1.00 & & 1.00 & & 1.00 & \\
\hline \multicolumn{15}{|l|}{ Region } \\
\hline Northeast & 20.89 & $14.36,29.38$ & 17.15 & $12.62,22.90$ & 14.38 & $10.44,19.49$ & 15.44 & $10.83,21.52$ & 0.65 & $0.52,0.81$ & 0.63 & $0.50,0.80$ & 0.67 & $0.48,0.94$ \\
\hline Midwest & 21.92 & $16.05,29.18$ & 28.00 & $22.48,34.29$ & 26.76 & $20.91,33.55$ & 28.98 & $22.70,36.18$ & 0.91 & $0.73,1.14$ & 1.03 & $0.83,1.27$ & 1.10 & $0.82,1.46$ \\
\hline South & 35.44 & $28.93,42.54$ & 30.26 & $25.18,35.88$ & 37.11 & $30.93,43.74$ & 33.93 & $27.69,40.78$ & 0.70 & $0.58,0.85$ & 0.91 & $0.76,1.08$ & 0.81 & $0.63,1.03$ \\
\hline West & 21.75 & $15.20,30.12$ & 24.58 & $19.08,31.07$ & 21.75 & $16.11,28.70$ & 21.66 & $16.21,28.31$ & 1.00 & & 1.00 & & 1.00 & \\
\hline
\end{tabular}

a $95 \%$ confidence interval.

b Unadjusted odds ratio.

design effects including clustered data. Cross tabulations were conducted with reckless drinking-related, chronic, and chronic/ severe categories of reckless drivers and sociodemographic variables and violent and non-violent antisocial behaviors. Multivariate multinomial logistic regression analyses were executed to assess the relationship of indicators of reckless driving to each psychiatric disorder while controlling for sociodemographic covariates and lifetime psychiatric diagnoses. Specifically, control variables used to reduce confounding included lifetime alcohol (alcohol abuse/ dependence) and drug (abuse/dependence on heroin, hallucinogens, cocaine/crack, marijuana, stimulants, painkillers, tranquilizers, and sedatives) use disorders, nicotine dependence, pathological gambling, and lifetime DSM-IV mood (major depression, dysthymia, and bipolar disorder) and anxiety (social phobia, generalized anxiety disorder, panic disorder, and specific phobia) disorders. Adjusted odds ratios (AORs) and 95\% confidence intervals are presented to reflect the strength of the associations. Adjusted odds ratios were considered statistically significant only if each odds ratio were accompanied by a confidence interval that did not include the value 1.0.

\section{Results}

\subsection{Sociodemographic characteristics across categories of reckless driving}

Table 1 displays sociodemographic characteristics of adults without a lifetime history of reckless driving compared to persons who reported a lifetime history of reckless driving/drinking related, chronically reckless, and chronic and severely reckless. The overall prevalence of engaging in any form of reckless driving over the lifecourse was $25.24 \%$. The prevalence of chronic/severe reckless driving (drivers license suspended or revoked) was $2.69 \%$. Compared to non-reckless drivers, those reporting a lifetime history of reckless driving were more likely to be men (reckless drinking $\mathrm{OR}=2.41,95 \% \mathrm{CI}=2.18,2.66$, chronic $\mathrm{OR}=4.02,95 \%$ $\mathrm{CI}=3.70,4.36$, chronic/severe $\mathrm{OR}=8.51, \mathrm{CI}=7.05,10.26$ ), born in the U.S. (reckless - drinking OR $=3.87,95 \% \mathrm{CI}=3.03,4.95$, chronic $\mathrm{OR}=2.45,95 \% \mathrm{CI}=2.02,2.96$, chronic/severe $\mathrm{OR}=2.10,95 \%$ $\mathrm{CI}=1.40,3.14)$, and were uniformly more likely to be younger in age. With respect to racial and ethnic differences, Whites and 
Native-Americans were significantly more likely to endorse all forms of reckless driving compared to Hispanics. African-Americans were significantly less likely $(\mathrm{OR}=0.71,95 \% \mathrm{CI}=0.56,0.90)$ to report episodic - drinking reckless driving. Asians were significantly less likely to report chronic $(\mathrm{OR}=0.60,95 \% \mathrm{CI}=0.44,0.83)$ and chronic/severe reckless driving $(\mathrm{OR}=0.33,95 \% \mathrm{CI}=0.15,0.73)$. Chronically reckless drivers were more likely to possess less than a high school education $(\mathrm{OR}=1.13,95 \% \mathrm{CI}=1.00,1.29)$ as were chronic/severe reckless drivers $(\mathrm{OR}=1.59,95 \% \mathrm{CI}=1.28,1.97)$ than non-reckless drivers. In contrast, the reckless - drinking category were less likely to possess lower levels of education. Few differences were observed with respect to income levels across categories although compared to non-reckless drivers, chronic/chronic and severe categories were more likely to earn in the $\$ 35,000-69,999$ range. Both chronic and chronic/severe categories of reckless drivers were more likely to be widowed/married/ divorced ( $\mathrm{OR}=1.28$ and 1.90 , respectively) than other categories. Finally, compared to persons from the western region of the U.S., individuals from the northeast were uniformly less likely to be reckless drivers across all categories. Respondents from the south were less likely to be reckless - drinking related drivers $(\mathrm{OR}=0.70$, $95 \% \mathrm{CI}=0.58,0.85$ ).

\subsection{Reckless driving and associated antisocial behaviors}

A consistent relationship was observed across the levels of reckless driving (see Table 2 ) with non-reckless drivers exhibiting the lowest rates of violent and non-violent antisocial behaviors, followed by the reckless/drinking-related and chronically reckless drivers, and chronic and severe reckless drivers reporting the highest levels. Specifically, the prevalence of antisocial behaviors was typically five-to-ten times greater (and sometimes more) for respondents reporting a lifetime history of chronic and severe reckless driving compared to respondents with no such history. The most prevalent non-violent behaviors in the chronic/severe category were staying out late $(58.69 \%, 95 \% \mathrm{CI}=55.28 \%, 62.03)$ and cutting or not attending class $(52.10 \%, 95 \% \mathrm{CI}=48.04 \%, 56.14 \%)$. Aggression and violent behaviors were also relatively prevalent in the group. For example, hitting someone so hard you could injure them $(27.43 \%, 95 \% \mathrm{CI}=24.42 \%, 30.65 \%)$, bullying or pushing others around $(22.70 \%, 95 \% \mathrm{CI}=19.76 \%, 25.94 \%)$, physically hurting others on purpose $(21.35 \%, 95 \% \mathrm{CI}=18.40 \%, 24.63 \%)$, and swapping blows with husband/wife or boyfriend/girlfriend $(20.02 \%, 95 \% \mathrm{CI}=17.34 \%$, $23.00 \%$ ). The least prevalent behaviors were forcing someone to have sex $(1.16 \%, 95 \% \mathrm{CI}=0.52 \%, 2.56 \%)$, robbing or mugging someone $(2.62 \%, 95 \% \mathrm{CI}=1.85 \%, 3.68 \%)$, and setting fires on purpose $(5.05 \%, 95 \% \mathrm{CI}=3.77,6.74)$.

\subsection{Multivariate multinomial logistic regression analysis examining associations between reckless driving and lifetime psychiatric comorbidity}

Table 3 summarizes results from multinomial logistic regression models that compared prevalence rates of lifetime psychiatric comorbidity for previously defined categories of reckless drivers with non-reckless drivers serving as the reference category. Recall that odds ratios are adjusted for sociodemographic factors (i.e., race, sex, education, marital status, age, income, region, and

Table 2

Prevalence of specific violent and non-violent antisocial behaviors among reckless drivers by severity of reckless driving history.

\begin{tabular}{|c|c|c|c|c|c|c|c|c|c|c|}
\hline \multirow[t]{2}{*}{ Behavior } & \multicolumn{2}{|c|}{$\begin{array}{l}\text { No Reckless Driving } \\
(N=33524)\end{array}$} & \multicolumn{2}{|c|}{$\begin{array}{l}\text { Episodic - drinking } \\
(N=3149)\end{array}$} & \multicolumn{2}{|c|}{ Chronic $(N=4212)$} & \multicolumn{2}{|c|}{$\begin{array}{l}\text { Chronic/Severe } \\
(N=1102)\end{array}$} & \multirow[t]{2}{*}{ Chi-Square } & \multirow[t]{2}{*}{$P$-value } \\
\hline & $\%$ & $\mathrm{CI}$ & $\%$ & $\mathrm{Cl}$ & $\%$ & $\mathrm{CI}$ & $\%$ & $\mathrm{CI}$ & & \\
\hline \multicolumn{11}{|l|}{ Violent } \\
\hline Force someone to have sex & 0.09 & $0.06,0.14$ & 0.15 & $0.07,0.31$ & 0.20 & $0.09,0.41$ & 1.16 & $0.52,2.56$ & 2.66 & 0.056 \\
\hline Get into lots of fights that you started & 1.38 & $1.22,1.56$ & 7.04 & $6.06,8.15$ & 6.86 & $5.94,7.92$ & 13.76 & $11.49,16.40$ & 41.82 & $<0.001$ \\
\hline Rob/mug someone or snatch a purse & 0.11 & $0.08,0.16$ & 0.59 & $0.37,0.95$ & 0.80 & $0.52,1.24$ & 2.62 & $1.85,3.68$ & 13.66 & $<0.001$ \\
\hline $\begin{array}{l}\text { Get into a fight that came to swapping blows } \\
\text { with Husband/Wife or Boyfriend/Girlfriend }\end{array}$ & 4.45 & $4.13,4.79$ & 13.42 & $12.08,14.88$ & 13.46 & $12.07,14.98$ & 20.02 & $17.34,23.00$ & 50.57 & $<0.001$ \\
\hline Use a weapon in a fight & 1.48 & $1.31,1.68$ & 6.13 & $5.11,7.34$ & 6.19 & $5.44,7.05$ & 11.65 & $9.58,14.08$ & 40.51 & $<0.001$ \\
\hline Hit someone so hard that you injure them & 2.99 & $2.73,3.27$ & 14.83 & $13.34,16.45$ & 15.12 & $13.77,16.58$ & 27.43 & $24.42,30.65$ & 60.96 & $<0.001$ \\
\hline Harass/threaten/blackmail someone & 0.74 & $0.63,0.87$ & 5.26 & $4.33,6.38$ & 4.19 & $3.47,5.05$ & 8.71 & $6.83,11.04$ & 33.25 & $<0.001$ \\
\hline Bully/push people & 3.56 & $3.29,3.85$ & 15.06 & $13.67,16.56$ & 14.23 & $13.03,15.53$ & 22.70 & $19.76,25.94$ & 57.78 & $<0.001$ \\
\hline Hurt an animal on purpose & 0.85 & $0.74,0.99$ & 5.24 & $4.35,6.30$ & 4.59 & $3.81,5.53$ & 7.62 & $5.88,9.83$ & 35.58 & $<0.001$ \\
\hline Physically hurt others on purpose & 2.66 & $2.43,2.92$ & 14.25 & $12.85,15.76$ & 10.71 & $9.64,11.88$ & 21.35 & $18.40,24.63$ & 51.37 & $<0.001$ \\
\hline \multicolumn{11}{|l|}{ Non-Violent } \\
\hline Set a fire on purpose & 0.48 & $0.39,0.61$ & 3.90 & $3.13,4.87$ & 2.50 & $1.97,3.17$ & 5.05 & $3.77,6.74$ & 25.67 & $<0.001$ \\
\hline Cut class, not go to class, leave without permission & 16.53 & $15.91,17.18$ & 39.01 & $36.97,41.09$ & 39.17 & $37.35,41.01$ & 52.10 & $48.04,56.14$ & 60.10 & $<0.001$ \\
\hline Stay out late at night & 19.83 & $19.03,20.66$ & 46.65 & $44.59,48.71$ & 43.23 & $41.35,45.13$ & 58.69 & $55.28,62.03$ & 62.84 & $<0.001$ \\
\hline Run away from home overnight & 3.61 & $3.35,3.90$ & 8.46 & $7.38,9.69$ & 10.61 & $9.32,12.05$ & 16.08 & $13.59,18.93$ & 42.63 & $<0.001$ \\
\hline Often absent from school & 4.46 & $4.18,4.76$ & 14.98 & $13.44,16.67$ & 13.77 & $12.42,15.25$ & 22.85 & $19.62,26.43$ & 42.82 & $<0.001$ \\
\hline Quit a job without knowing where to find another & 8.06 & $7.59,8.55$ & 22.13 & $20.46,23.89$ & 23.31 & $21.74,24.95$ & 32.66 & $29.41,36.09$ & 57.35 & $<0.001$ \\
\hline More than once quit a school program without a plan & 2.60 & $2.31,2.93$ & 8.09 & $6.95,9.41$ & 7.23 & $6.27,8.32$ & 11.07 & $9.21,13.25$ & 28.45 & $<0.001$ \\
\hline Travel around without a plan & 2.02 & $1.84,2.23$ & 7.56 & $6.47,8.82$ & 8.18 & $7.18,9.30$ & 11.79 & $9.55,14.47$ & 41.33 & $<0.001$ \\
\hline Have no regular place to live & 1.58 & $1.40,1.78$ & 5.87 & $4.93,6.98$ & 6.91 & $6.02,7.91$ & 10.09 & $7.92,12.75$ & 36.72 & $<0.001$ \\
\hline Live with others at least 1 month & 7.98 & $7.40,8.59$ & 21.21 & $19.43,23.10$ & 21.94 & $20.33,23.64$ & 27.11 & $23.65,30.88$ & 55.16 & $<0.001$ \\
\hline Lie a lot & 3.12 & $2.88,3.38$ & 12.61 & $11.23,14.13$ & 11.25 & $10.10,12.51$ & 19.81 & $16.97,22.99$ & 49.51 & $<0.001$ \\
\hline Use a false or made up name/alias & 1.25 & $1.11,1.41$ & 5.39 & $4.58,6.34$ & 4.61 & $3.73,5.69$ & 7.58 & $5.79,9.87$ & 29.30 & $<0.001$ \\
\hline Scam/con someone for money & 0.63 & $0.52,0.75$ & 4.21 & $3.48,5.09$ & 3.69 & $2.94,4.63$ & 9.25 & $7.22,11.78$ & 28.26 & $<0.001$ \\
\hline Destroy others' property & 1.52 & $1.35,1.71$ & 12.31 & $10.91,13.86$ & 9.18 & $8.13,10.34$ & 17.54 & $14.64,20.87$ & 47.21 & $<0.001$ \\
\hline Fail to pay off your debts & 2.31 & $2.09,2.56$ & 8.98 & $7.85,10.26$ & 10.09 & $8.93,11.37$ & 17.95 & $15.15,21.14$ & 42.80 & $<0.001$ \\
\hline Steal anything from others & 4.80 & $4.40,5.23$ & 26.30 & $24.43,28.26$ & 20.49 & $18.92,22.15$ & 27.68 & $24.49,31.11$ & 58.73 & $<0.001$ \\
\hline Forge someone's signature & 1.17 & $1.01,1.36$ & 6.07 & $5.12,7.17$ & 4.70 & $3.68,5.98$ & 7.36 & $5.73,9.40$ & 28.44 & $<0.001$ \\
\hline Shoplift & 6.59 & $6.14,7.08$ & 30.91 & $28.70,33.22$ & 23.52 & $21.76,25.38$ & 34.76 & $31.18,38.53$ & 52.22 & $<0.001$ \\
\hline Make money illegally & 0.90 & $0.77,1.05$ & 8.30 & $7.16,9.60$ & 8.21 & $7.22,9.33$ & 16.38 & $13.64,19.55$ & 41.50 & $<0.001$ \\
\hline Do something you could have been arrested for & 7.36 & $6.86,7.89$ & 51.73 & $49.43,54.01$ & 35.61 & $33.73,37.54$ & 52.31 & $48.33,56.27$ & 69.29 & $<0.001$ \\
\hline
\end{tabular}


Table 3

Lifetime prevalence and multivariate multinomial logistic regression analysis examining associations between reckless driving and lifetime psychiatric comorbidity.

\begin{tabular}{|c|c|c|c|c|c|c|c|c|c|c|c|c|c|c|}
\hline \multirow[t]{2}{*}{ Comorbid Psychiatric Disorder } & \multicolumn{2}{|c|}{$\begin{array}{l}\text { No Reckless } \\
\text { Driving } \\
(N=33524)\end{array}$} & \multicolumn{2}{|c|}{$\begin{array}{l}\text { Episodic - } \\
\text { drinking related } \\
(N=3149)\end{array}$} & \multicolumn{2}{|c|}{$\begin{array}{l}\text { Chronic } \\
(N=4212)\end{array}$} & \multicolumn{2}{|c|}{$\begin{array}{l}\text { Chronic/severe } \\
(N=1102)\end{array}$} & \multicolumn{2}{|c|}{$\begin{array}{l}\text { Episodic - } \\
\text { drinking related } \\
\text { AOR }\end{array}$} & \multicolumn{2}{|c|}{ Chronic AOR } & \multicolumn{2}{|c|}{$\begin{array}{l}\text { Chronic/severe } \\
\text { AOR }\end{array}$} \\
\hline & $\%$ & $\mathrm{CI}$ & $\%$ & $\mathrm{CI}$ & $\%$ & $\mathrm{CI}$ & $\%$ & $\mathrm{CI}$ & $95 \%$ & $\mathrm{CI}$ & $95 \%$ & $\mathrm{CI}$ & $95 \%$ & $\mathrm{CI}$ \\
\hline Nicotine dependence & 12.92 & $12.17,13.70$ & 38.37 & $36.25,40.54$ & 31.62 & $29.73,33.57$ & 39.67 & $35.82,43.66$ & 1.46 & $1.28,1.66$ & 1.39 & $1.24,1.55$ & 1.44 & $1.17,1.77$ \\
\hline Marijuana use disorder & 4.08 & $3.79,4.40$ & 26.20 & $24.56,27.90$ & 19.57 & $17.99,21.26$ & 33.09 & $29.55,36.84$ & 1.86 & $1.60,2.16$ & 1.59 & $1.37,1.85$ & 2.12 & $1.73,2.61$ \\
\hline \multicolumn{15}{|l|}{ Any alcohol use disorder } \\
\hline Abuse/Dependence & 18.84 & $17.75,19.97$ & 81.53 & 79.77,83.17 & 60.96 & $58.93,62.96$ & 73.98 & $70.51,77.18$ & 10.25 & $8.90,11.81$ & 3.64 & $3.27,4.04$ & 4.80 & $3.91,5.90$ \\
\hline Amphetamine & 0.73 & $0.61,0.88$ & 6.36 & $5.45,7.40$ & 5.65 & $4.69,6.79$ & 9.98 & $7.78,12.72$ & 1.04 & $0.75,1.45$ & 1.61 & $1.15,2.26$ & 1.34 & $0.86,2.09$ \\
\hline Opioid & 0.56 & $0.45,0.70$ & 4.38 & $3.55,5.39$ & 3.83 & $3.11,4.71$ & 7.06 & $5.42,9.14$ & 0.96 & $0.62,1.47$ & 1.11 & $0.72,1.71$ & 0.74 & $0.46,1.20$ \\
\hline Sedative & 0.34 & $0.27,0.44$ & 3.63 & $2.95,4.46$ & 2.89 & $2.30,3.62$ & 6.55 & $4.96,8.60$ & 1.19 & $0.71,1.98$ & 1.15 & $0.67,1.98$ & 1.32 & $0.67,2.56$ \\
\hline Tranquilizer & 0.31 & $0.24,0.41$ & 3.26 & $2.59,4.09$ & 2.49 & $1.95,3.17$ & 6.48 & $4.92,8.50$ & 1.14 & $0.62,2.09$ & 0.94 & $0.51,1.74$ & 1.42 & $0.68,2.99$ \\
\hline Inhalant/Solvent & 0.09 & $0.06,0.14$ & 1.28 & $0.89,1.86$ & 0.88 & $0.59,1.32$ & 1.81 & $1.10,2.98$ & 1.08 & $0.52,2.26$ & 0.91 & $0.40,2.04$ & 0.66 & $0.30,1.47$ \\
\hline Cocaine & 1.04 & $0.91,1.19$ & 8.78 & $7.68,10.02$ & 6.95 & $6.03,7.99$ & 17.10 & $14.13,20.56$ & 1.32 & $1.02,1.71$ & 1.32 & $1.01,1.74$ & 2.49 & $1.79,3.47$ \\
\hline Hallucinogen & 0.61 & $0.49,0.75$ & 6.12 & $5.17,7.24$ & 4.06 & $3.27,5.03$ & 9.15 & $7.14,11.66$ & 0.97 & $0.67,1.41$ & 0.83 & $0.53,1.29$ & 0.90 & $0.59,1.39$ \\
\hline Heroin & 0.06 & $0.04,0.10$ & 0.58 & $0.36,0.94$ & 0.50 & $0.31,0.80$ & 1.85 & $1.09,3.11$ & 2.23 & $0.90,5.56$ & 1.64 & $0.63,4.28$ & 3.31 & $0.94,11.71$ \\
\hline \multicolumn{15}{|l|}{ Mood disorder } \\
\hline Major depressive disorder & 14.69 & $14.00,15.41$ & 27.19 & $25.42,29.04$ & 22.65 & $21.15,24.22$ & 22.91 & $20.05,26.05$ & 1.16 & $1.02,1.32$ & 1.21 & 1.07,1.37 & 1.11 & $0.88,1.39$ \\
\hline Bipolar disorder & 4.26 & $3.96,4.59$ & 10.88 & $9.76,12.11$ & 10.71 & $9.42,12.17$ & 12.28 & $10.23,14.67$ & 0.92 & $0.76,1.11$ & 1.15 & $0.94,1.41$ & 0.96 & $0.72,1.29$ \\
\hline Dysthymia & 3.65 & $3.37,3.95$ & 7.77 & $6.73,8.96$ & 6.15 & $5.31,7.11$ & 6.77 & $5.20,8.78$ & 0.95 & $0.74,1.22$ & 0.92 & $0.74,1.15$ & 0.95 & $0.65,1.38$ \\
\hline \multicolumn{15}{|l|}{ Anxiety disorder } \\
\hline Panic disorder & 3.47 & $3.20,3.76$ & 7.41 & $6.43,8.53$ & 5.63 & $4.77,6.63$ & 5.31 & $3.71,7.55$ & 1.20 & $0.98,1.46$ & 1.15 & $0.92,1.45$ & 1.02 & $0.65,1.58$ \\
\hline Social phobia & 4.14 & $3.79,4.52$ & 10.88 & $9.64,12.25$ & 6.84 & $5.90,7.93$ & 6.83 & $5.15,8.99$ & 1.34 & 1.08,1.67 & 0.93 & $0.77,1.12$ & 0.88 & $0.59,1.31$ \\
\hline Specific phobia & 8.48 & $7.91,9.08$ & 16.48 & $14.82,18.28$ & 11.63 & $10.34,13.05$ & 11.97 & $10.01,14.26$ & 1.25 & $1.06,1.46$ & 1.08 & $0.94,1.25$ & 1.09 & $0.84,1.41$ \\
\hline Generalized anxiety & 3.56 & $3.24,3.92$ & 7.56 & $6.49,8.79$ & 6.17 & $5.25,7.24$ & 5.15 & $3.77,7.01$ & 1.00 & $0.78,1.27$ & 1.05 & $0.83,1.31$ & 0.76 & $0.49,1.17$ \\
\hline Conduct disorder & 1.01 & $0.88,1.15$ & 1.23 & $0.82,1.84$ & 1.44 & $1.00,2.07$ & 1.27 & $0.67,2.38$ & 0.72 & $0.42,1.23$ & 0.89 & $0.59,1.34$ & 0.75 & $0.38,1.49$ \\
\hline Family history of antisocial behavior & 19.17 & $18.18,20.21$ & 41.56 & $39.38,43.76$ & 31.62 & $29.87,33.43$ & 39.42 & $35.81,43.14$ & 1.73 & $1.54,1.96$ & 1.30 & $1.17,1.45$ & 1.68 & $1.41,2.00$ \\
\hline Psychotic disorder & 0.63 & $0.53,0.75$ & 1.49 & $1.07,2.07$ & 1.28 & $0.91,1.80$ & 1.41 & $0.81,2.45$ & 0.89 & $0.55,1.43$ & 0.83 & $0.51,1.34$ & 0.59 & $0.28,1.26$ \\
\hline \multicolumn{15}{|l|}{ Personality disorder } \\
\hline Avoidant & 1.90 & $1.71,2.11$ & 4.34 & $3.62,5.18$ & 4.19 & $3.43,5.12$ & 3.62 & $2.45,5.31$ & 0.99 & $0.70,1.41$ & 1.19 & $0.88,1.60$ & 0.74 & $0.42,1.32$ \\
\hline Dependent & 0.39 & $0.32,0.49$ & 0.89 & $0.60,1.33$ & 0.73 & $0.43,1.25$ & 1.39 & $0.73,2.64$ & 0.77 & $0.39,1.53$ & 0.64 & $0.33,1.22$ & 1.13 & $0.43,2.98$ \\
\hline Obsessivecompulsive & 6.41 & $5.97,6.87$ & 14.92 & $13.46,16.49$ & 12.99 & $11.69,14.41$ & 14.25 & $11.93,16.94$ & 1.25 & $1.06,1.48$ & 1.27 & $1.08,1.49$ & 1.21 & $0.92,1.60$ \\
\hline Paranoid & 3.37 & $3.09,3.68$ & 7.57 & $6.51,8.79$ & 8.49 & $7.47,9.62$ & 11.41 & $9.11,14.20$ & 1.03 & $0.81,1.30$ & 1.35 & $1.07,1.70$ & 1.56 & $1.07,2.29$ \\
\hline Schizoid & 2.47 & $2.24,2.73$ & 5.73 & $4.83,6.77$ & 5.43 & $4.66,6.32$ & 7.06 & $5.40,9.17$ & 1.07 & $0.84,1.38$ & 1.04 & $0.81,1.32$ & 1.11 & $0.80,1.55$ \\
\hline Antisocial & 1.35 & $1,20,1.52$ & 12.30 & $11.01,13.73$ & 10.03 & $8.90,11.29$ & 18.26 & $15.58,21.27$ & 2.90 & $2.30,3.64$ & 2.50 & $2.01,3.10$ & 3.35 & $2.54,4.42$ \\
\hline Histrionic & 1.19 & $1.06,1.35$ & 4.50 & $3.77,5.37$ & 3.67 & $2.92,4.59$ & 5.76 & $4.45,7.44$ & 1.33 & $0.99,1.79$ & 1.21 & $0.90,1.63$ & 1.45 & $0.98,2.13$ \\
\hline
\end{tabular}

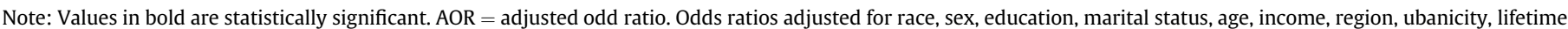

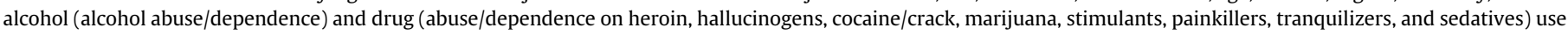

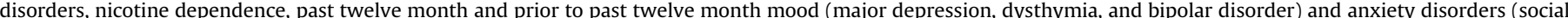

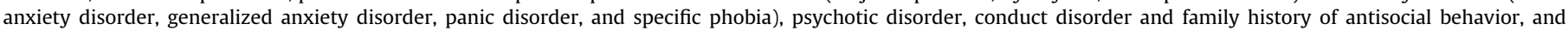
personality disorder.

urbanicity), previously described lifetime DSM-IV psychiatric disorders including substance use disorders, and family history of antisocial behavior. With respect to substance use disorders, there were significant findings across nicotine dependence, marijuana use disorder, alcohol use disorder and cocaine dependence for all categories of reckless driving with strongest effects observed for alcohol use disorder; specifically, (AOR $=10.25,95 \% \mathrm{CI}=8.90$, 11.81 ) for reckless-drinking, $(\mathrm{AOR}=3.64,95 \% \mathrm{CI}=3.27,4.04)$, for chronic reckless, and ( $\mathrm{AOR}=4.80,95 \% \mathrm{CI}=3.91,5.90)$ for chronic/ severe reckless drivers. Chronic/severe reckless drivers were approximately two-and-one-half times more likely to have a cocaine use disorder $(\mathrm{AOR}=2.49,95 \% \mathrm{CI}=1.79,3.47)$. Chronic reckless drivers were significantly more likely to have an amphetamine use disorder (AOR $=1.61,95 \% \mathrm{CI}=1.15,2.26)$. Although heroin dependence was associated with increased likelihood for reckless driving, the effects were not statistically significant due to low cell sizes.

Small effects were found for mood and anxiety disorders for the reckless-alcohol and chronic categories. Major depression was elevated for both of the categories (AOR $=1.16,95 \% \mathrm{CI}=1.02,1.32)$ and $(\mathrm{AOR}=1.21,95 \% \mathrm{CI}=1.07,1.37)$. Reckless-drinking drivers were at increased risk for social $(\mathrm{AOR}=1.34,95 \% \mathrm{CI}=1.08,1.67)$ and specific $(A O R=1.25,95 \% \mathrm{CI}=1.06,1.46)$ phobia. No significant findings were found for the chronic/severe category. All three reckless driver groups were more likely to have a family history of antisocial behavior than non-reckless drivers. With respect to personality disorders, reckless-drinking drivers were more likely to possess a diagnosis of obsessive-compulsive personality $(\mathrm{AOR}=1.25,95 \% \mathrm{CI}=1.06,1.48)$ and antisocial personality disorder $($ AOR $=2.90,95 \% \mathrm{CI}=2.30,3.64)$. Chronic reckless drivers were more likely to have a diagnosis of obsessive-compulsive (AOR $=1.27,95 \% \mathrm{CI}=1.08,1.49$ ), paranoid (AOR $=1.35,95 \%$ $\mathrm{CI}=1.07,2.29)$, and antisocial $(\mathrm{AOR}=2.50,95 \% \mathrm{CI}=2.01,3.10)$ personality disorders. Chronic/severe reckless drivers were more likely to be diagnosed with paranoid $(\mathrm{AOR}=1.56,95 \% \mathrm{CI}=1.07$, 2.29) and antisocial ( $\mathrm{AOR}=3.35,95 \% \mathrm{CI}=2.54,4.42$ ) personality disorders.

\section{Discussion}

To our knowledge, this is the largest national epidemiological study examining the association between forms of reckless driving and comorbid behavioral and mental health conditions. With respect to sociodemographic patterns, the current investigation found that young men living with lower levels of educational attainment were at increased odds of being reckless drivers. Men in general, were over eight times more likely than women to report having had their drivers license suspended or revoked. 
Interestingly, persons born in the U.S. were over twice as likely as persons born outside of the U.S. to report reckless driving. This finding suggests that there may be something about American culture that promotes reckless driving or that persons moving to the U.S from other countries are simply less likely to report reckless driving due to fear of American law enforcement. Of course, this type of social desirability bias could extend to other variables in the study as well.

There was support for our first hypothesis that reckless driving would be positively associated with externalizing behaviors. The convergent validity for reckless driving indicators was strong. Reckless driving was significantly associated with numerous and varied forms of antisocial behavior including getting into numerous physical altercations, bullying, property destruction, lying, cruelty to animals, stealing, and harassment. Results indicate that the prevalence of antisocial behaviors among episodic-drinking and chronic reckless drivers was much higher than among non-reckless drivers and the prevalence rate among chronic/severe reckless drivers were much higher than these two forms. The second hypothesis, that severity of reckless driving whereby persons who have had their licenses suspended or revoked (chronic/severe reckless drivers) would be associated with greater intensity of these externalizing behaviors in controlled multivariate analyses was partially supported. Marijuana and cocaine use disorder along with antisocial personality disorder were elevated in this group. Family history of antisocial behavior was relatively similar across categories. Alcohol use disorder, however, had its strongest effects in association with the episodic-drinking related category. Together these findings demonstrate the problematic nature of not only reckless driving generally but that reckless driving that leads to license suspension and revocation is part and parcel of a relatively severe antisocial behavior syndrome (Gottfredson and Hirschi, 1990; Hirschi and Gottfredson, 1994). Clearly, greater policy emphasis on expanding comprehensive treatment of substance use disorders and psychiatric comorbidities can potentially function as a universal and selective prevention strategy for the reduction of reckless driving episodes.

Despite recognition that alcohol and substance use are associated with reckless driving, there has been a relative lack of attention paid to antisocial propensity and reckless driving. Sensation seeking, impulsivity, and disregard for other persons are features of persons who often violate the rights of others throughout the life-course. The implications of these findings suggest that policies that attenuate criminal careers and aggressive behaviors may in turn have a powerful effect on reducing reckless driving (DeLisi, 2005). Persons with a history of alcohol dependence have been shown to display increased levels of antisocial and aggressive behaviors and obtained more traffic violations than those individuals who did not display these personality traits (Zelhart, 1972), which is consistent with higher rates of antisocial behaviors in those individuals who had been arrested for drinking and driving (Lapham et al., 2001; Gottfredson and Hirschi, 1990; Argeriou et al., 1985; Junger and Tremblay, 1999). Prior research reported that those with fewer traffic citations had personalities characterized by social apprehensiveness, increased levels of emotional stability and self-sufficiency, and lower levels of impulsiveness, whereas those with greater traffic citations and motor vehicle accidents had increased levels of emotional instability, irritability, impulsiveness, sensation seeking, increased aggression, resentment, low frustration tolerance, oversensitivity to criticism, depression, decreased levels of assertiveness, and perception that one is unable to control one's future (Donovan et al., 1983).It is also important to note that about $75 \%$ of those who have been arrested for driving under the influence of liquor had prior involvement with the criminal justice system (Argeriou et al., 1985). This finding shows the overlap between drinking and driving and the general propensity toward antisocial behavior.

\subsection{Limitations}

Study results should be interpreted in light of several limitations. First, given that the study data are cross-sectional, temporal ordering of variables does not permit firm conclusions regarding causal determinants. Reported findings cannot clarify the etiologic relationship between forms of reckless driving and its correlates. For example, the use and abuse of alcohol may be associated with reckless driving due to its disinhibiting effects on neuroregulatory processes that facilitate executive governance in the face of risky behaviors. Further, the associations with antisocial behavior may be part of a general externalizing propensity to engage in the use and abuse of various substances. We can suggest firmly that reckless driving and externalizing psychopathology are intertwined. Prospective designs over longer swaths of time are needed to untangle the dynamics of specific externalizing behaviors and reckless driving. Another limitation is that the NESARC excludes persons under age 18 and therefore relies on retrospective respondent recall of reckless driving and other behaviors over potentially long periods of time. Underreporting or biased reporting with younger respondents recalling better than older respondents is quite possible. An additional limitation is the wording of the first item used to define reckless driving-drinking which lacked specificity and could be capturing mere risk-taking propensity. However, the adjusted analyses did substantiate the relationship of the category to possessing an alcohol use disorder. Despite these limitations, study findings offer new and important epidemiologic insights into the costly problem of reckless driving and its correlates in the United States. Finally, greater policy emphasis on expanding comprehensive treatment of substance use disorders and psychiatric comorbidities can potentially function as a universal and selective prevention strategy for the reduction of reckless driving episodes.

\section{Role of the funding source}

None declared.

\section{Contributors}

Michael Vaughn conceptualized the study, led the literature review and study design, and analytic strategy. Qiang Fu conducted the statistical analysis. Matt DeLisi, Kevin Beaver, Rebecca Define, Brian Perron, and Matthew Howard reviewed literature and contributed writing.

\section{Conflict of interest}

None declared.

\section{Acknowledgement}

NESARC was funded by the National Institute on Alcohol Abuse and Alcoholism with additional support provided by the National Institute on Drug Abuse. The authors are grateful for support from NIH grants: DA021405 and K07CA104119. The contents of the article are solely the responsibility of the authors and do not necessarily represent the official view of the National Institutes of Health.

\section{References}

Argeriou M, McCarty M, Blacker E. Criminality among individuals arraigned for drinking and driving in Massachusetts. J Stud Alc 1985;46:525-30.

Blanchard EB, Hickling EJ, Taylor AE, Loos W. Psychiatric morbidity associated with motor vehicle accidents. J Nerv Ment Disease 1995;183:495-504. 
Blanco C, Grant J, Petry NM, Simpson HB, Alegria A, Liu S-M, et al. Prevalence and correlates of shoplifting in the United States: results from the national epidemiologic survey on alcohol and related conditions (NESARC). Am J Psych 2008;165:905-13.

Blincoe L, Seay A, Zaloshnja T, Miller T, Romano E, Luchter S, et al. The economic impact of motor vehicle crashes, 2000. U.S. Department of Transportation, National Highway Traffic Safety Administration; 2002. pp. 1-94.

Borges G, Cherpitel CJ, Mondragón L, Poznyak V, Peden M, Guiterrez I. Episodic alcohol use and risk of nonfatal injury. Am J Epidemiol 2004;159:565-71.

Centers for Disease Control and Prevention. Motor vehicle safety page. Available at: http://www.cdc.gov/motorvehiclesafety/index.html [accessed 14.10.09].

De Vries APJ, Kassam-Adams N, Cnaan A, Sherman-Slate E, Gallagher PR Winston FK. Posttraumatic stress disorder in children and parents after pediatric traffic injury. Pediatrics 1999;104:1293-9.

DeLisi M. Career criminals in society. Thousand Oaks, CA: Sage; 2005.

Donovan DM, Marlatt GA. Personality subtypes among driving - while-intoxicated offenders: relationship to drinking behavior and driving risk. J Consult and Clin Psych 1982;50:241-9.

Donovan DM, Marlatt GA, Salzberg PM. Drinking behavior, personality factors and highrisk driving: a review and theoretical formulation. J Stud Alc 1983;44:395-428.

Goldstein RB, Grant BF, Ruan WJ, Smith SM, Saha TD. Antisocial personality disorder with childhood- vs. adolescence-onset conduct disorder: results from the national epidemiologic survey on alcohol and related conditions. J of Nerv Ment Disease 2006;194:667-75.

Gottfredson MR, Hirschi T. A general theory of crime. Stanford, CA: Stanford University Press; 1990.

Grant BF, Harford T, Dawson DA, Chou PS, Pickering RP. The alcohol use disorder and associated disabilities interview schedule (AUDADIS): reliability of alcohol and drug modules in a general population sample. Drug Alc Dep 1995;39:37-44.

Grant BF, Dawson DA, Stinson FS, Chou PS, Kay W, Pickering R. The alcohol use disorde and associated disabilities interview schedule-IV (AUDADIS-IV): reliability of alcohol consumption, tobacco use, family history of depression and psychiatric diagnostic modules in a general population sample. Drug Alc Dep 2003;71:7-16.

Grant BF, Hasin D, Stinson FS, Dawson DA, Chou SP, Ruan WJ, et al. Prevalence correlates, and disability of personality disorders in the United States: results from the national epidemiologic survey on alcohol and related conditions. J Clin Psychiatry 2004a;65:948-58.
Grant BF, Stinson FS, Dawson DA, Chou SP, Ruan WJ, Pickering RP. Co-occurrence of 12-month alcohol and drug use disorders and personality disorders in the United States: results from the national epidemiologic survey on alcohol and related conditions. Arch Gen Psychiatry 2004b;61:361-8.

Hasin D, Carpenter KM, McCloud S, Smith M, Grant BF. The alcohol use disorders and associated disabilities interview schedule (AUDADIS): reliability of alcohol and drug modules in a clinical sample. Drug Alc Dep 1997;44:133-41.

Junger M, Tremblay RE. Self-control, accidents, and crime. Crim Just Behav 1999;26:485-501.

Kelly E, Darke S, Ross J. A review of drug use and driving: epidemiology, impairment, risk factors and risk perceptions. Drug Alc Review 2004;23:319-44.

Keppel-Benson JM, Ollendick TH, Benson MJ. Post-traumatic stress in children following motor vehicle accidents. J Child Psych Psychiatry 2002;43:203-12.

Krug EG, Sharma GK, Lozano R. The global burden of injuries. Am J Pub Health 2000;90:523-6.

Lapham SC, Smith E, Baca J, Baca JC, Chang I, Skipper BJ, et al. Prevalence of psychiatric disorders among persons convicted of driving while impaired. Arch Gen Psychiatry 2001;58:943-9.

Nabi H, Consoli SM, Chastang JF, Chiron M, Lafont S, Lagarde E. Type A behavior pattern, risky driving behaviors, and serious road traffic accidents: a prospective study of the GAZEL Cohort. Am J Epidemiol 2005;161:864-70.

Research Triangle Institute. Software for survey data analysis, SUDAAN. Version 9.0. [Computer software]. Research Triangle Park, NC: Research Triangle Institute; 2004.

Hirschi T, Gottfredson MR, editors. The generality of deviance. New Brunswick, NJ: Transaction; 1994

Trends in Alcohol Related Fatalities. Persons killed, by highest driver blood alcohol concentration (BAC) in the Crash, 1994-2008-State: USA. Available at: http:// www-fars.nhtsa.dot.gov/Trends/TrendsAlcohol.aspx [accessed 16.10.09].

Ursano RJ, Fullerton CS, Epstein RS, Crowley B, Kao T-C, Vance K, et al. Acute and posttraumatic stress disorder in motor vehicle accident victims. Am J Psych 1999; 156:589-95.

Villaveces A, Cummings P, Koepsell TD, Rivara FP, Lumley T, Moffat J. Association of alcohol-related laws with deaths due to motor vehicle and motorcycle crashes in the United States, 1980-1997. Am J Epidemiol 2003;157:131-40.

Zelhart PF. Types of alcoholics and their relationship to traffic violations. J Stud Alc 1972;33:811-3. 